Acknowledgements: I have no acknowledgements to declare.

Disclosure of Interests: None declared

DOI: 10.1136/annrheumdis-2021-eular.1420

\section{AB0425 1 CLINICAL AND PATHOLOGICAL FEATURES OF BREAST CANCER IN PATIENTS WITH SYSTEMIC SCLEROSIS: PRELIMINARY DATA FROM THE SCLERO-BREAST STUDY}

A. Spinella ${ }^{1}$, A. Toss $^{2}$, C. Isca ${ }^{2}$, C. Vacchi ${ }^{1}$, A. Iannone ${ }^{3}$, L. Magnani ${ }^{4}$, P. Castrignanò $^{1}$, M. De Pinto ${ }^{1}$, C. Laura ${ }^{2}$, A. Maiorana $^{5}$, C. Salvarani ${ }^{1,4}$, M. Dominici ${ }^{2}$, D. Giuggioli ${ }^{1}$. ${ }^{1}$ University of Modena and Reggio Emilia, Scleroderma Unit, Rheumatology Unit, Modena, Italy; ${ }^{2}$ University of Modena and Reggio Emilia, Department of Oncology and Hematology, Modena, Italy; ${ }^{3}$ University of Modena and Reggio Emilia, Department of Surgery, Medicine, Dentistry and Morphological Sciences with Transplant Surgery, Oncology and Regenerative Medicine Relevance, Modena, Italy; ${ }^{4}$ AUSL-IRCCS of Reggio Emilia, Italy, Rheumatolgy, Reggio Emilia, Italy; ${ }^{5}$ University of Modena and Reggio Emilia, Pathology Unit, Modena, Italy

Background: Systemic Sclerosis (SSc) is a rare and life-threatening connective tissue disease characterized by vascular dysfunction, specific autoimmune abnormalities and fibrosis of the skin and internal organs. Previous studies have shown a 1.5-fold increase in cancer risk in SSc patients compared with the general population, including breast cancer $(\mathrm{BC})$. The relationship between $\mathrm{BC}$ and SSc has long been discussed but past research has been contradictory and inconclusive on this topic.

Objectives: The aim of our project was to analyze clinical and pathological characteristics of BC developed by SSc subjects and possible correlations with scleroderma features. Here we present the preliminary data from the Sclero-Breast study.

Methods: Our observational retrospective multicenter study enrolled 33 SSc women with a personal history of BC identified at two Rheumatology/SSc Units in the north of Italy between January 2017 and December 2019 (Ic/dcSSc 23/9, 1 unknown; mean age at SSc onset 57 years, range 32-73). All patients underwent general and instrumental assessment: smoking habits; presence of skin ulcers, calcinosis, teleangectasia; presence of gastro-intestinal and kidney involvement; interstitial lung disease (at HR-CT); pulmonary function tests; ECG abnormalities; echocardiographic assessment of pulmonary arterial hypertension (PAH); videocapillaroscopic pattern; autoantibody profile; exposure to immunosuppressive and vasoactive therapies; status at last follow-up evaluation and cause of death. Clinical and pathological characteristics of BC were also evaluated: age at diagnosis; menopausal status; histotype; hormone receptor status; MIB1, HER2 expression; clinical and pathological stage at diagnosis; metastatic sites; type of loco-regional treatment (surgery and radiotherapy); type of systemic treatment (neoadjuvant/adjuvant chemotherapy and endocrine treatment); other cancers and time from diagnosis of the first disorder to the second one.

Results: A total of $54.5 \%$ of subjects developed BC before SSc (median interval of 5 years), whereas $45.5 \%$ of patients developed BC after SSc (median delay of 8 years). $54.5 \%$ of patients showed interstitial lung disease and the cause of death of the 6 deceased subjects was $\mathrm{PAH}$. A significant association $(p<0.05)$ was observed between the use of immunosuppressive therapy and diffuse skin extension, negative ACA, positive Anti-Scl-70 and interstitial lung disease, but not with BC status. $93.1 \%$ of patients were diagnosed with an early-stage tumor, $70.8 \%$ of invasive carcinomas with a low MIB-1, $8.3 \%$ with a tubular histotype, while $42.8 \%$ presented with a Luminal A-like tumor. $66.6 \%$ underwent breast conserving surgery and $55.5 \%$ RT after surgery. $40 \%$ of patients developed interstitial lung disease after RT and $20 \%$ dcSSc.

Conclusion: According to our preliminary data, SSc patients developed BC at good prognosis, suggesting a de-escalation strategy of cancer therapies. On these grounds, a proper screening is mandatory in order to allow for early cancer detection in SSc patients. Further investigations on larger numbers of patients are needed. First of all, they would further clarify the intriguing relationship between BC and SSc. Secondly, they would help to explore the common biological and molecular pathways at the basis of these two disorders, with the aim to improve $\mathrm{BC}$ diagnosis and prognosis and to personalize oncological targeted treatments in this subset of fragile patients.

Disclosure of Interests: None declared

DOI: 10.1136/annrheumdis-2021-eular.1450
AB0426

CANCER SCREENING IN IDIOPATHIC INFLAMMATORY MYOPATHIES: TEN YEARS EXPERIENCE FROM A SINGLE CENTER

E. Trallero-Araguás ${ }^{1}$, A. Gil-Vila ${ }^{2}$, X. Martínez-Gómez ${ }^{3}$, M. Alvarado-Cardenas ${ }^{2}$, M. Simo-Perdigó ${ }^{4}$, J. Ros ${ }^{5}$, I. Pinal-Fernandez ${ }^{6}$, A. Selva-O'callaghan7. ${ }^{1}$ Vall d'Hebron Hospital, Rheumatology, BARCELONA, Spain; ${ }^{2}$ Vall d'Hebron Hospital, Medicine, Barcelona, Spain; ${ }^{3}$ Vall d'Hebron Hospital, Preventive Medicine and Epidemiology, Barcelona, Spain; ${ }^{4}$ Vall d'Hebron Hospital, Nuclear Medicine, Barcelona, Spain; ${ }^{5}$ Vall d'Hebron Hospital, Medical Oncology, Barcelona, Spain; ${ }^{6}$ National Institutes of Health, Rheumatology, Bethesda, Spain; ${ }^{2}$ Vall d'Hebron Hospital, Medicine, Barcelona, Spain

Background: There is a well-recognized association between cancer and myositis, so cancer screening at diagnosis is recommended.

Objectives: We aim to report the results of our cancer screening strategy and to ascertain the reliability of using PET/CT to identify cancer-associated myositis (CAM) in a large cohort of patients with myositis from a single center over 10 years.

Methods: This retrospective observational study included all patients diagnosed with any type of myositis except for inclusion body myositis. Cancer screening strategy was individualized according to clinical and serological data, including $\mathrm{PET} / \mathrm{CT}$ as the main test to detect occult cancer (OC). Procedures derived from a positive PET/CT were registered. Qualitative data expressed as percentages, and quantitative data as the median with the interquartile range were analyzed. A ROC curve was used to estimate the reliability of PET/CT for CAM diagnosis. Results: Seventy-seven out of 131 patients underwent a PET/CT for OC screening. The performance of the PET/CT in patients with myositis at disease onset yielded an area under the curve ROC of 0.87 (0.73-0.97) for CAM diagnosis. Invasive procedures in 7 (9\%) patients without a final diagnosis of cancer did not cause derived complications. Patients not evaluated for OC did not develop cancer after a median follow-up of 3.3 years (1.7-6.7).

Conclusion: Cancer screening strategy should be individualized. PET/CT at myositis onset seems to be an efficient approach to rule out CAM. This practice does not seem to significantly increase harm to patients related to the additional tests needed to clarify inconclusive results.

Disclosure of Interests: None declared

DOI: 10.1136/annrheumdis-2021-eular.1457

\section{AB0427 \\ ANTI-NOR90 AUTOANTIBODIES: FAVORABLE OR UNFAVORABLE PROGNOSIS?}

C. Valero $^{1}$, J. P. Baldivieso ${ }^{1}$, I. Llorente ${ }^{1}$, E. F. Vicente-Rabaneda ${ }^{1}$, L. Esparcia Pinedo. $^{2}$, R. Garcia de Vicuna ${ }^{1}$, M. A. Alfranca ${ }^{2}$, S. Castañeda ${ }^{1} .{ }^{1}$ Hospital Universitario de la Princesa, Rheumatology, Madrid, Spain $;{ }^{2}$ Hospital Universitario de la Princesa, Immunology, Madrid, Spain

Background: Anti-NOR 90 autoantibodies (anti-NOR90 Ab) are autoantibodies that target nucleolar transcription factor 1 or hUBF, involved in transcription of RNA polymerase I. These autoantibodies have been detected in $6.1 \%$ of patients with Systemic Sclerosis (SSc), but their clinical or prognostic significance has not been clearly defined. Anti-NOR90 Ab have been mostly associated with limited scleroderma with mild organ involvement and can also be found in other rheumatic diseases such as rheumatoid arthritis, systemic lupus erythematosus or Sjogren's syndrome.

Objectives: The aim of this study was to identify the main clinical characteristics of patients with positive anti-NOR90 in our Centre.

Methods: This is a retrospective, descriptive, cross-sectional study of all patients with positive anti-NOR90 Ab between January 2013 and December 2020 in a single center. Autoantibodies testing was performed using Euroimmun EUROLINE SSc profile IgG autoAb assay kit. Patient demographics, clinical characteristics, associated diagnoses, laboratory and immunological findings were collected. Results: We identified a total of 26 patients with at least a positive value for antiNOR90 Ab (Table 1). In most cases anti-NOR90 patients were ANA positive, predominantly with nucleolar pattern and coexisted with other SSc autoantibodies. 12 patients had rheumatic diseases and two had SSc, both with limited cutaneous SSc and absence of organ involvement. 14 patients had no definite diagnosis. Clinical features of anti-NOR90 patients are represented in Figure 1. Five patients presented Raynaud's phenomenon, two cases with pathological nailfold capillaroscopy and one patient had SSc. There was no patient with skin ulcers, calcinosis, interstitial lung disease or pulmonary hypertension. Four patients had gastroesophageal reflux disease and one patient presented antral vascular ectasia. Six patients developed some neoplasm. 